\title{
Suppression of soft nuclear bremsstrahlung in proton-nucleus collisions
}

\author{
M.J. van Goethem ${ }^{1}$, L. Aphecetche ${ }^{2, a}$, J.C.S. Bacelar ${ }^{1}$, H. Delagrange ${ }^{2, a}$, J. Díaz ${ }^{3}$, \\ D. d'Enterria ${ }^{2, a}$, M. Hoefman ${ }^{1}$, R. Holzmann ${ }^{4}$, H. Huisman ${ }^{1}$, N. Kalantar-Nayestanaki ${ }^{1}$, \\ A. Kugler ${ }^{5}$, H. Löhner ${ }^{1}$, G. Martínez ${ }^{2, a}$, J.G. Messchendorp ${ }^{1}$, R.W. Ostendorf ${ }^{1}$, S. \\ Schadmand $^{1, b}$, R.H. Siemssen ${ }^{1}$, R.S. Simon ${ }^{4}$, Y. Schutz ${ }^{2, a}$, R. Turrisi ${ }^{1, c}$, M. Volkerts ${ }^{1}$, \\ V. Wagner ${ }^{5}$, and H.W. Wilschut ${ }^{1}$ \\ ${ }^{1}$ Kernfysisch Versneller Instituut, Zernikelaan 25, NL-9747 AA Groningen, The Netherlands \\ ${ }^{2}$ Grand Accélérateur National d'Ions Lourds, F-14076 Caen Cedex 5, France \\ ${ }^{3}$ Institut de Física Corpuscular, E-46100 Burjassot, Spain \\ ${ }^{4}$ Gesellschaft für Schwerionenforschung, D-64291 Darmstadt, Germany \\ ${ }^{5}$ Nuclear Physics Institute, 25068 Řež u Prahy, Czech Republic
}

(November 3, 2018)

\begin{abstract}
Photon energy spectra up to the kinematic limit have been measured in 190 $\mathrm{MeV}$ proton reactions with light and heavy nuclei to investigate the influence of the multiple-scattering process on the photon production. Relative to the predictions of models based on a quasi-free production mechanism a strong suppression of bremsstrahlung is observed in the low-energy region of the photon spectrum. We attribute this effect to the interference of photon amplitudes due to multiple scattering of nucleons in the nuclear medium.
\end{abstract}

PACS numbers: 13.75.-n; 13.40.-f; 24.10.Cn; 25.40.Ep

Typeset using REVTEX 
In collisions between nucleons electromagnetic radiation can be emitted due to the rapid change in the nucleon velocity (bremsstrahlung). Accordingly, in nucleus-nucleus collisions bremsstrahlung is emitted due to the individual collisions of the constituent nucleons. Earlier experiments with protons and heavy ions [1] indicated that bremsstrahlung is dominantly produced in first-chance proton-neutron collisions. Consequently, dynamical nuclear reaction models include photon production in the incoherent quasi-free collision limit, i.e., free nucleon-nucleon (NN) bremsstrahlung cross sections are employed assuming on-shell nucleons and the intensities of the individual scattering processes are added rather than their amplitudes.

At sufficiently high energies multiple-scattering processes become important in reactions of protons with nuclei. A significant effect on the radiation process is expected due to the influence of multiple scattering and off-shell propagation of nucleons. In this case, the bremsstrahlung amplitudes from different steps in the scattering process interfere and, therefore, the individual bremsstrahlung contributions may not be added incoherently. This so called LPM effect was predicted by Landau, Pomerančuk, and Migdal [2] for the successive Coulomb scattering of electrons in matter, resulting in a reduced bremsstrahlung rate once the mean free path is shorter than the coherence length. This suppression has been reported for pair creation from cosmic-ray photons [3], and for bremsstrahlung from high-energy electrons [四] in accelerator experiments. The general importance of coherence effects on particle production and absorption in (non-)equilibrium dense matter has been discussed in the literature [5] [7]. Such effects are, e.g., relevant for soft photon and dilepton production in hot hadronic matter [8,9]. However, no quantitative analysis for the LPM effect in nuclear bremsstrahlung has been reported so far.

To study the influence of the nuclear medium on the bremsstrahlung spectrum we have measured the energy spectra and angular distributions of photons up to the kinematic limit in reactions of $190 \mathrm{MeV}$ protons with a range of targets. A strong suppression of bremsstrahlung relative to a quasi-free production model is observed in the low-energy regime of the photon spectrum. The present study was part of the experimental program with the 
photon spectrometer TAPS [10,11] at the AGOR facility of the KVI Groningen. A proton beam with a typical intensity of $0.5-2 \mathrm{nA}$ was incident on solid targets of $\mathrm{Au}, \mathrm{Ag}, \mathrm{Ni}$, and $\mathrm{C}$ with thicknesses ranging from 20 to $56 \mathrm{mg} / \mathrm{cm}^{2}$. External conversion of photons was kept below $1 \%$ by the use of a $70 \mathrm{~cm}$ diameter carbon-fibre scattering chamber with $4 \mathrm{~mm}$ wall thickness.

The photon spectrometer TAPS was configured in 6 blocks of $64 \mathrm{BaF}_{2}$ crystals each at a distance of $66 \mathrm{~cm}$ from the target. The setup covered the polar angular range between $57^{\circ}$ and $176^{\circ}$ on both sides of the beam with an azimuthal acceptance of $-21^{\circ}<\phi<21^{\circ}$. The granularity of the TAPS setup resulted in an angular resolution of $5.2^{\circ}$. Photons were separated from nuclear particles via their time-of-flight with respect to the radiofrequency signal (RF) of the cyclotron. The time resolution was about 1 ns (FWHM). In addition, pulse-shape discrimination was employed. The event trigger required an energy deposition of at least $5 \mathrm{MeV}$ in $\mathrm{BaF}_{2}$ module. The signals from the plastic veto detectors in front of the $\mathrm{BaF}_{2}$ scintillators were used to select photons and protons on the trigger level. The relative energy calibration was determined from the characteristic energy deposited by cosmic-ray muons. The absolute calibration was provided by the $\pi^{0}$ mass peak and the $15.1 \mathrm{MeV}$ photons originating from inelastic proton scattering on ${ }^{12} \mathrm{C}$. A small residual background from cosmic-ray muons within the trigger gate was removed by subtracting the photon spectrum obtained by gating on a random time window with respect to the RF.

Two-photon invariant mass spectra from events with two coincident photons were analysed in order to obtain the $\pi^{0}$ decay contribution. The raw $\pi^{0}$ distributions were corrected for the finite acceptance and the response of TAPS. The measured pion distribution was extrapolated by Monte-Carlo simulations [12,13] into regions of missing acceptance by analyzing the angular distributions in small energy bins of $2 \mathrm{MeV}$. The spectrum of photons from $\pi^{0}$ decay peaks at about $70 \mathrm{MeV}$ with a yield at least a factor 5 below the inclusive photon yield. After correction for contributions from $\pi^{0}$ decay, photon spectra at laboratory polar angles of $75^{\circ}, 115^{\circ}$ and $155^{\circ}$ in a window of $\pm 5^{\circ}$ are obtained with a systematic uncertainty of $5 \%$. Uncertainties due to beam current and target thickness contribute another 
$5 \%$. Here we present the results near $90^{\circ}$ in order to minimize the influence of the reference frame.

Fig. 1 shows a compilation of the photon spectra at a lab. angle of $75^{\circ}$ for the four targets studied here. The double differential cross sections have been normalized to the geometrical cross section $\sigma_{r}=1.44 \pi A^{2 / 3} \mathrm{fm}^{2}$ of each reaction with target mass number $A$. The spectra extend up to the kinematic limit $E_{\max }=T_{C M}+\mathrm{Q}$, where $\mathrm{Q}$ is the Q-value of the reaction and $T_{C M}$ the center-of-mass energy. If plotted as function of the scaled photon energy $E_{\gamma} / E_{\max }$, all data in Fig. 1 above $100 \mathrm{MeV}$ fall on the same curve [12,14]. The shape of the photon spectra displays a plateau between 30 and $80 \mathrm{MeV}$ and an exponential decrease towards the kinematic limit. This shape is different from photon spectra in heavy ion reactions [15,16], where nearly exponential slopes have been observed above $30 \mathrm{MeV}$. The rise at photon energies below $30 \mathrm{MeV}$ for the heavier targets can be attributed to statistical photon emission.

For comparison with dynamical model calculations including the multiple-scattering process we employ the Intra-Nuclear Cascade (INC) code of Cugnon [17]. The INC model was chosen because it reproduces well many aspects of proton-nucleus reactions at these bombarding energies [18]. Furthermore it allows the study of photon-nucleon correlations [19] because it conserves correlations between scattered particles. This is a necessary feature for the inclusion of the NN bremsstrahlung production in a non-perturbative manner with the kinematically correct $p n \gamma$ process using the Soft-Photon Approximation (SPA) for free $p n$ bremsstrahlung [20]. We found that the optimal way to implement Pauli blocking in INC was achieved by requiring all final scattering states to lie above the Fermi surface. The nucleon phase-space distributions from the INC calculations agree well with those obtained using the Boltzmann-Uehling-Uhlenbeck (BUU) [21] transport model. The BUU results describe the photon spectrum $\left(E_{\gamma}>30 \mathrm{MeV}\right)$ from $180 \mathrm{~A} \mathrm{MeV} \mathrm{Ar+Ca} \mathrm{collisions} \mathrm{fairly} \mathrm{well}$ [16] although slightly overpredicting the yield on the soft side of the spectrum.

Fig. 2 shows the photon spectrum for the $\mathrm{Au}$ target at $75^{\circ}$ in comparison with the INC and BUU results. Both models agree quite well with each other in the full spectrum but 
overestimate significantly the experimental photon yield at low and intermediate photon energies $\left(\mathrm{E}_{\gamma} \leq 100 \mathrm{MeV}\right)$. Good agreement between the experimental data and the calculations is found in the hard part of the spectrum, even near the kinematic limit. The multiple-step contribution from INC has been indicated separately in Fig. 2. The overall agreement between experiment and theory would be much better if no multiple scattering was taken into account. It seems as if multiple-scattering processes are overestimated in theory. However, the amount of multiple scattering in INC has been checked against the experimental proton yields at large angles. These proton yields, in which multiple-scattering processes are essential, agree within the error margins for all targets studied [12]. Therefore, multiple scattering is well described.

In the nuclear medium the nucleon mean free path is $\lambda_{m f p}=1 /\left(\rho \cdot \sigma_{N N}\right) \approx 2 \mathrm{fm}$, based on an average NN scattering cross section $\sigma_{N N}=30 \mathrm{mb} 22$ at $190 \mathrm{MeV}$ and the nuclear saturation density $\rho=0.16 \mathrm{fm}^{-3}$. Therefore, nuclear bremsstrahlung can be quenched for a photon wavelength $\lambda \geq \lambda_{m f p}$ or a photon energy $E_{\gamma} \leq \hbar c / \lambda_{m f p} \approx 90 \mathrm{MeV}$. The strength of quenching of course increases with decreasing photon energy. In a simplified model based on the classical description of bremsstrahlung production in hard collisions we have estimated the analytical shape of the LPM effect in a two-step $p+$ nucleus reaction [12]. Each segment of the proton trajectories defines a production amplitude with a definite relative phase and therefore must be added coherently. The time between two collisions is characterized by the mean collision time $\tau=\lambda_{m f p} /\left(g \beta_{0} c\right)=\tau_{0} / g$ with $\beta_{0}$ the incoming proton velocity, i.e. $\tau_{0} \approx 4 \mathrm{fm} / \mathrm{c}$. The factor $g$ takes into account that in subsequent collisions the velocity of the leading particle is reduced. A value $g \approx 0.5$ is expected to describe the mean time between the first and second collision, i.e., $\tau \approx 8 \mathrm{fm} / \mathrm{c}$. Averaging over the time distribution $(1 / \tau) \exp (-t / \tau)$ we derive the following quenching factor, whose analytical form is motivated by several theoretical calculations [5,9,23]:

$$
f_{q}=\xi\left(1-\frac{\alpha}{1+\left(\frac{E_{\gamma}}{\hbar} \tau\right)^{2}}\right) .
$$

The parameter $\alpha$ is related to the fraction of energy remaining for the leading particle in 
subsequent collisions [12], i.e., $\alpha \approx 1 / g^{2} \approx 0.25 . \xi$ is an overall scaling factor. The INC calculation was adjusted in an ad hoc manner to account for medium effects by multiplying the spectrum obtained from INC with the quenching factor $f_{q}$ from Eq. (11).

The experimental data were fitted with the product $I N C \cdot f_{q}$, where $I N C$ represents the full INC spectrum and $\alpha, \xi$ and $\tau$ are free parameters. For the spectra obtained at three different angles of $75^{\circ}, 115^{\circ}$ and $155^{\circ}$ we find $\alpha \approx 1$ and a mean collision time $\tau=2.4 \pm 0.6$ $\mathrm{fm} / \mathrm{c}$ for the $\mathrm{Ni}, \mathrm{Ag}$ and $\mathrm{Au}$ targets and $3.7 \pm 0.5 \mathrm{fm} / \mathrm{c}$ for the $\mathrm{C}$ target. These values for the collision time are much smaller than the expected average time interval between two hard NN collisions in nuclei ( $8 \mathrm{fm} / \mathrm{c}$, see above). From this observation one must conclude that hard NN collisions alone are insufficient to explain the quenching of soft photons. Other effects likely to increase the observed collision frequency (reduced parameter $\tau$ ) may be multiple soft collisions, but also a modification of the elementary photon production process in the nuclear medium. The latter hypothesis is supported by the observation that the dipole contribution expected from the elementary proton-neutron angular distribution appears to be absent in the reactions studied here, as was observed also elsewhere [24]. In our data we observe the corresponding result from the fact that $\xi$ increases from $\xi=0.96 \pm 0.06$ at $75^{\circ}$ to $\xi=4.3 \pm 2.0$ at $155^{\circ}$.

The separation between dynamical effects and LPM quenching is complicated due to the partitioning of the NN interaction in the nuclear medium into a mean-field and a collision component in the models. The available dynamical models are all of semiclassical nature and our new data indicate the need to include consistently the medium modifications and the interference phenomena. The development of a quantum transport theory for photon production in intermediate-energy proton+nucleus reactions, was already started in ref. [25] but did not go beyond the conventional quasi-particle approximation, i.e., the correlations and off-shell nucleon propagation in the medium were not taken into account. A new approach has been taken up recently [7]. The in-medium photon production cross section is calculated from a microscopic NN interaction including the spectral width of the baryon propagators. Two sources of multiple-scattering effects can be identified: one of minor im- 
portance is scattering of final state nucleons before or after photon radiation; more significant appears to be the multiple scattering during off-shell nucleon propagation before the photon is emitted. Preliminary results [26] for a $200 \mathrm{MeV} p+$ nucleus reaction indicate that this approach leads to a remarkable suppression of soft photons below $80 \mathrm{MeV}$, in qualitative agreement with our data. This approach requires, however, a non-zero temperature of the target nucleons. Recently, another theoretical approach [27 was taken where the kinetic equations that determine the evolution of the two-particle Green's function in matter were derived in the transport approximation for soft-photon production. The correlations in the medium allow multiple scattering to occur without requiring multiple hard collisions, thus yielding Eq. (1) with $f_{q}\left(\alpha=1, \tau=\tau_{0}\right)$, i.e. $g=1$, in agreement with the empirical result. We thus obtain an energy dependence of the photon spectrum with the functional form

$$
f_{E} \sim \frac{E_{\gamma}}{E_{\gamma}^{2}+\left(\hbar / \tau_{0}\right)^{2}(Z / N)^{2}} \cdot \beta_{0}^{2} \cdot\left(1-E_{\gamma} / E_{\max }\right)
$$

This spectrum incorporates the factor $1-E_{\gamma} / E_{\max }$ to describe the kinematic limit which is absent in the soft photon approach. (The precise form of this limit may vary, cf. [24].) The proton to neutron ratio $(\mathrm{Z} / \mathrm{N})$ describes the reduced quenching observed in heavy nuclei. The absolute cross section is determined by the geometrical reaction cross section. The suppression of soft photons can be described quantitatively as shown in Fig. 3 by the dark lines. The amount of quenching can be seen from comparison with the result where the collision frequency (i.e., $\hbar / \tau_{0}$ ) is set to zero (the grey dotted lines). This approaches the quasi-free result of the INC model. Eq. (2) describes also well the published data 14,24,28 for $168 \mathrm{MeV} \mathrm{p}+\mathrm{Tb}$ and $145 \mathrm{MeV} \mathrm{p}+\mathrm{Pb}$ by only adjusting $\beta_{0}$ according to the respective beam energy. This shows that also at lower beam energy quenching occurs, but the effect at photon energies above $30 \mathrm{MeV}$ is small and went unnoticed so far.

In summary, new experimental data have been presented for nuclear bremsstrahlung from the soft-photon region up to the kinematic limit in proton+nucleus reactions. We observe a strong suppression of the soft bremsstrahlung cross section in comparison with the prediction of transport models that include bremsstrahlung on basis of quasi-free nucleon- 
nucleon collisions. Applying a phenomenological quenching factor appropriate for sequential hard collisions between nucleons, we can fit the data using the average collision time as a free parameter. We find that its value is much shorter than expected on basis of the collision times in a transport model. New theoretical models are being developed using simplified reaction dynamics but taking into account medium effects such as nucleon correlations and off-shell propagation of the nucleons involved in the photon production. An analytical form for the bremsstrahlung production in nuclear matter based on these assumptions was obtained which describes the suppression of soft bremsstrahlung. These results show the importance of multiple-scattering processes beyond the classical picture of multiple hard collisions.

The effort of the AGOR team in providing high-quality beam is gratefully acknowledged. We thank the KVI theory group and A. Korchin, A.V. Koshelkin, and A. Sedrakian for valuable discussions and for providing results from their calculations prior to publication. This work was supported in part by FOM, the Netherlands, by IN2P3 and CEA, France, BMBF and DFG, Germany, DGICYT and the Generalitat València, Spain, by GACR, Czech Republic, and by the European Union HCM network contract HRXCT94066. 


\section{REFERENCES}

a present address: Laboratoire SUBATECH, BP 20722, F-44307 Nantes Cedex 3, France.

b present address: II. Physikalisches Institut, Universität Gießen, D-35392 Gießen, Germany.

c present address: Dipartimento di Fisica e INFN, I-35131 Padova, Italy.

[1] H. Nifenecker, Prog. in Part. Nucl. Phys. 23 (1989) 271

[2] L.D. Landau and I. Pomerančuk, Dokl. Akad. Nauk SSSR 92 (1953) 553; A.B. Migdal, Sov. Phys. JETP 5 (1957) 527

[3] J. Benisz, Z. Chylinski, W. Wolter, Nuovo Cim. XI, 4 (1959) 525

[4] S. Klein, Rev. Mod. Phys. 71 (1999) 1501

[5] J. Knoll and D.N. Voskresensky, Phys. Lett. B 351 (1995) 43 and Ann. Phys. (NY) 249 (1996) 532

[6] A.V. Koshelkin, Phys. Rev. C 59 (1999) 936

[7] A. Sedrakian and A.E.L. Dieperink, Phys. Lett. B 463 (1999) 145, and private communication

[8] E.V. Shuryak, Phys. Lett. B 231 (1989) 175

[9] J. Cleymans, V.V. Goloviznin, and K. Redlich, Phys. Rev. D 47 (1993) 173

[10] H. Ströher, Nuclear Physics News 6, 7 (1996)

[11] A.R. Gabler et al., Nucl. Instr. Meth. A 346, 168 (1994)

[12] M.J. van Goethem, PhD thesis, Univ. Groningen 2000

[13] L. Aphecetche et al., Phys. Lett. B 519 (2001) 8

[14] M. Kwato Njock et al., Phys. Lett. B 207 (1988) 269 
[15] Y. Schutz et al., Nucl. Phys. A 622 (1997) 404

[16] G. Martínez et al., Phys. Lett. B 461 (1999) 28

[17] J. Cugnon, Nucl. Phys. A 489 (1988) 781

[18] J. Cugnon et al., Nucl. Phys. A 620 (1997) 475

[19] M.J. van Goethem et al., to be published

[20] A.Yu. Korchin and O. Scholten, Phys. Rev. C 58 (1998) 1098

[21] W. Cassing et al. Phys. Rep. 188 (1990) 363

[22] J. Franz et al., Nucl. Phys. A 490 (1988) 667

[23] T. Alm et al., Phys. Rev. C 52 (1995) 1972

[24] J. Clayton et al., Phys. Rev. C 45 (1992) 1815

[25] K. Nakayama and G.F. Bertsch, Phys. Rev. C 40 (1989) 2520

[26] A. Sedrakian, private communication

[27] A.V. Koshelkin, private communication

[28] J.A. Pinston et al., Phys. Lett. B 218 (1989) 128 


\section{FIGURES}

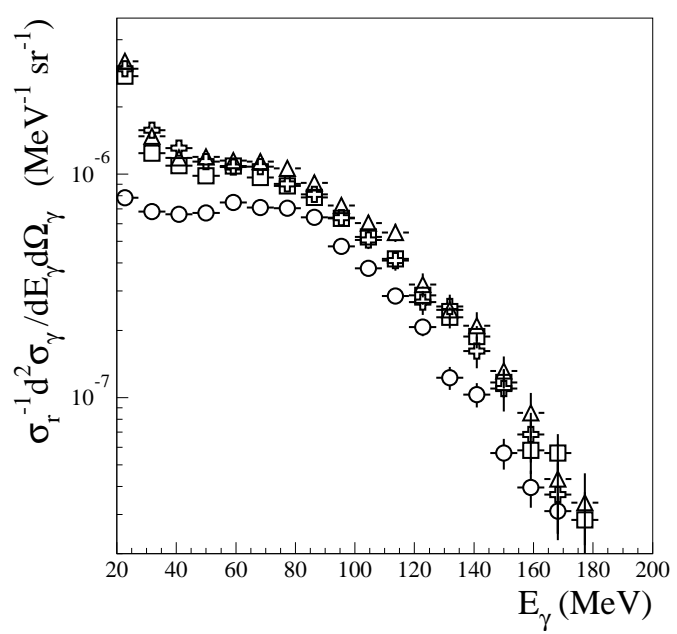

FIG. 1. Target-mass dependence of photon spectra for $190 \mathrm{MeV}$ protons on $\mathrm{C}$ (circles), Ni (triangles), Ag (squares), and Au (crosses) targets at a lab. angle of $75^{\circ}$. The double differential cross sections have been normalized to the geometrical reaction cross section.

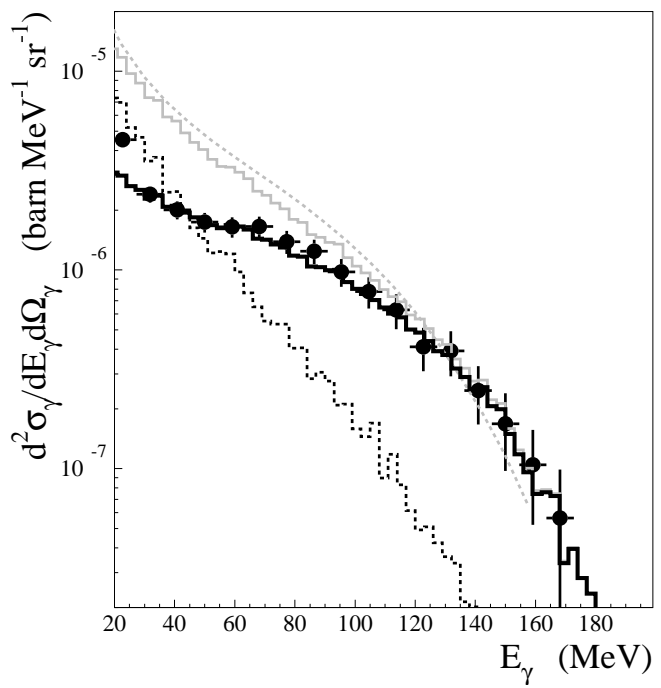

FIG. 2. Photon spectrum for $190 \mathrm{MeV} \mathrm{p}+\mathrm{Au}$ at an angle of $75^{\circ}$ (filled circles), compared to results of the INC (grey histogram) and BUU (dashed line) models. The dashed histogram shows the multiple-step contribution from the INC model. The black histogram is the INC result multiplied with the quenching factor $f_{q}$ from Eq. (1). 


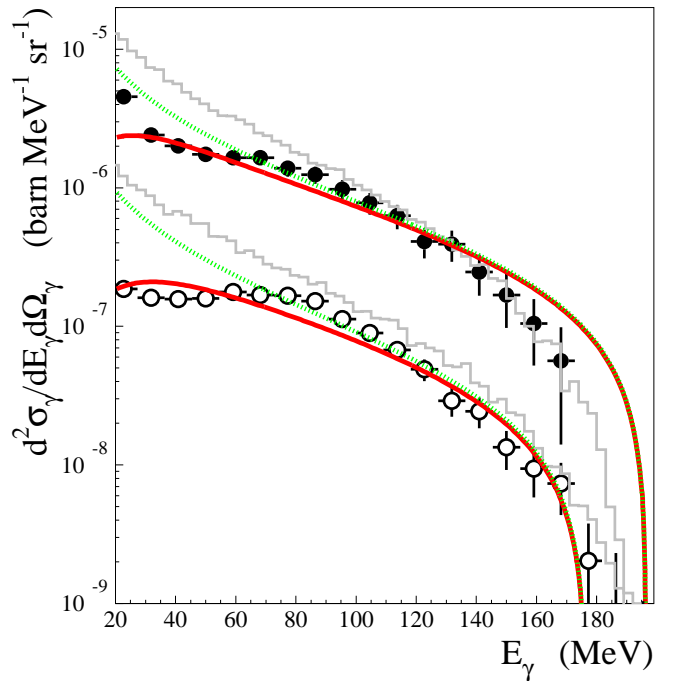

FIG. 3. Photon spectrum at a lab. angle of $75^{\circ}$ for $190 \mathrm{MeV} \mathrm{p}+\mathrm{Au}$ (top, filled circles) and $\mathrm{p}+\mathrm{C}$ (bottom, circles). The grey histograms indicate the results from the INC model. The dark lines are obtained using Eq. (2), the dotted grey lines correspond to Eq. (2) with the collision frequency set to zero. 